\title{
METABOLISM OF ATP BY TESTIS MITOCHONDRIA OF 25-DAY-OLD RATS
}

\author{
MANNFRED A. HOLLINGER \\ Department of Pharmacology, School of Medicine, University of California, \\ Davis, California 95616, U.S.A.
}

(Received 14th September 1970)

\begin{abstract}
Summary. Mitochondrial fraction from rat testes was found to metabolize ATP at a faster rate and to a more complete extent than mitochondria from liver or kidney. Catabolism of ATP by rat testis mitochondria is essentially complete by $30 \mathrm{~min}$. Both ADP and AMP are formed in equal amounts.
\end{abstract}

ATP is known to play a key rôle in the storage and utilization of metabolic energy in most mammalian tissues. The enhancement of testicular protein biosynthesis by glucose appears to be related to the production of ATP (Means \& Hall, 1968). However, there does not appear in the literature any information regarding the metabolism of this essential nucleotide in the testes. This communication presents preliminary data relating to the catabolism of ATP by a mitochondrial fraction derived from rat testes.

The animals used in these experiments were 25-day-old male SpragueDawley rats. Testes were removed, decapsulated, pooled and homogenized with a motor driven teflon pestle (clearance 0.1 to $0.15 \mathrm{~mm}$ ) in $0.32 \mathrm{M}$-sucrose $10: 1(\mathrm{v} / \mathrm{w})$. The homogenization was carried out in a cold room at $4^{\circ} \mathrm{G}$. The homogenate was centrifuged at $3000 \mathrm{~g}$ for $10 \mathrm{~min}$ and the sediment discarded. The supernate was then centrifuged at $10,000 \mathrm{~g}$ for $20 \mathrm{~min}$ to obtain the mitochondrial fraction. The mitochondrial pellet was resuspended in distilled water and the protein concentration determined (Lowry, Rosebrough, Farr \& Randall, 1951).

Incubation of the mitochondrial fraction with appropriate co-factors and radioactive ATP was carried out as described elsewhere (Hollinger, 1970). The nucleotides were separated by paper and thin-layer chromatography in a solvent system containing butanol, acetone, glacial acetic acid, water and concentrated ammonium hydroxide $(14: 10: 6: 9: 1, \mathrm{v} / \mathrm{v}) . \mathrm{R}_{\mathrm{f}}$ values for the migration of ATP, ADP and AMP in this solvent system are $0.15,0.28$ and 0.52 , respectively. The UV-absorbing spots corresponding to the migration of authentic samples were cut out and assayed for radioactivity. Recovery of radioactivity ranged from 89 to $95 \%$.

The mitochondria were fixed for $1 \mathrm{hr}$ in $1.6 \%$ glutaraldehyde in $0.1 \mathrm{M}$ phosphate buffer at $4^{\circ} \mathrm{C}$. They were post-fixed in $1 \% \mathrm{OsO}_{4}$, dehydrated in ethanol, and embedded in Epon 812. Ultra-thin sections were cut with diamond 
TABLE 1

COMPARISON OF ATP- $8{ }^{14} \mathrm{C}$ CATABOLISM BY THE MITOGHONDRIAL FRACTION DERIVED FROM RAT TESTIS, LIVER AND KIDNEY

\begin{tabular}{l|c|c|c}
\hline \multicolumn{1}{c|}{ Tissue } & $A T P$ & $A D P$ & \multicolumn{1}{c}{$A M P$} \\
\hline Testis & $6 \cdot 8 \pm 2 \cdot 7$ & $47 \cdot 9 \pm 3 \cdot 6$ & $45 \cdot 6 \pm 6 \cdot 3$ \\
Liver & $63 \cdot 0 \pm 2 \cdot 1$ & $31 \cdot 2 \pm 2 \cdot 7$ & $8 \cdot 8 \pm 0 \cdot 6$ \\
Kidney & $27 \cdot 0 \pm 4 \cdot 1$ & $35 \cdot 0 \pm 1 \cdot 0$ & $37 \cdot 5 \pm 3 \cdot 3$ \\
\hline
\end{tabular}

Animals were 25 days of age. Assay performed as described in text. Each value represents the mean \% of total radioactivity recovered \pm S.E. remaining after 30 -min incubation.

knives on a Sorvall Porter-Blum MT-2 Ultramicrotome. They were stained with uranyl acetate saturated in $50 \%$ ethanol, and lead citrate. They were examined and photographed in an AEI EM-801 electron microscope.

Table 1 presents a comparison of ATP catabolism in the crude mitochondrial fraction derived from prepubertal rat testis, liver and kidney. ATP catabolism was found to differ with the tissue. After 30-min incubation, far less of the total radioactivity recovered was present as ATP in the testis compared to the liver and kidney. This would appear to suggest a very active ATPase system in rat testis. The presence of relatively high levels of labelled ADP and AMP formed from the testis further suggests that ATP catabolism by the crude mitochondrial fraction is more complete in the testis than in the liver or kidney and therefore phosphatase activity catalyzing dephosphorylation of ADP is presumably higher.

Text-figure 1 presents the catabolic time course for ATP in the crude mitochondrial fraction from rat testis over a period of $50 \mathrm{~min}$. During this range of

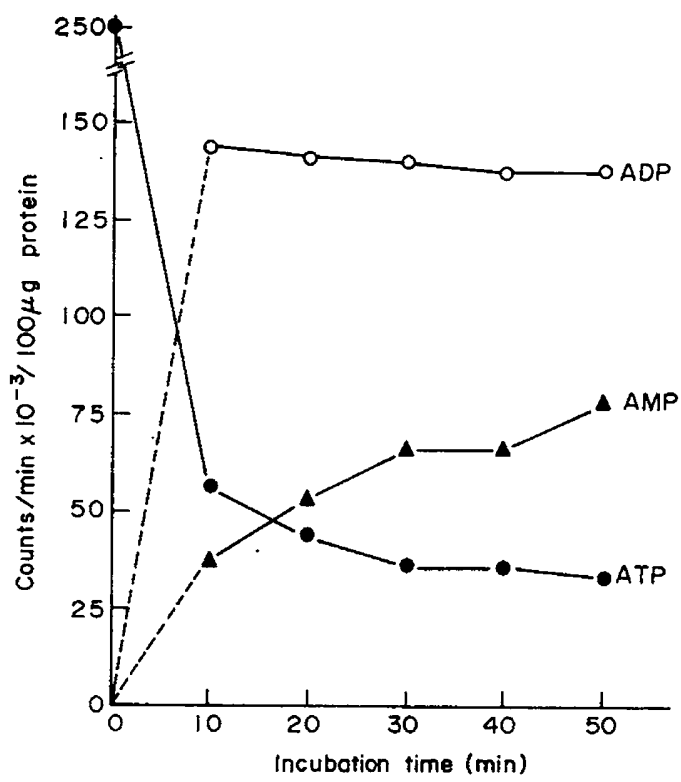

TexT-PIC. 1. Effects of incubation time on ATP-8 $-{ }^{14} \mathrm{C}$ catabolism by rat testis mitochondrial fraction. Each point is the mean of duplicate determinations. 


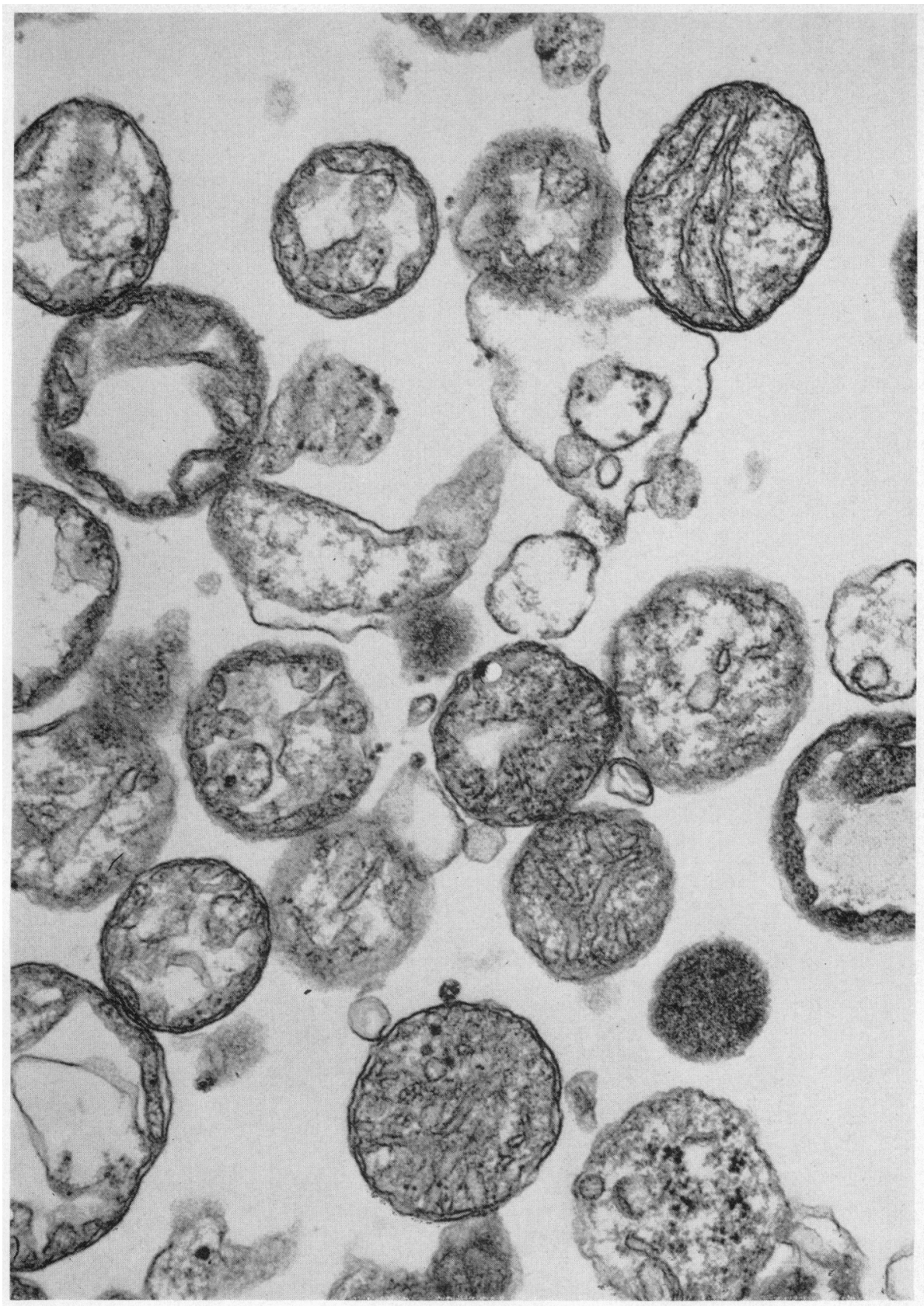

Electron photomicrograph of mitochondrial fraction obtained from 25-day-old rat testes. $\times 140,000$. 
time, ATP metabolism occurs throughout the incubation period, being greatest during the first $10 \mathrm{~min}$. In addition, ADP formation is maximal at $10 \mathrm{~min}$, gradually decreasing thereafter. The level of AMP progressively increases with incubation time.

Plate 1 presents an electron photomicrograph of the mitochondrial fraction used in this study. There was relatively little contamination by other cellular components. Although some appeared swollen or contained vacuoles, the majority appeared morphologically normal.

Previous studies in vitro dealing with endogenous ATP levels in whole rat testis tissue have observed a significantly rapid decline in the concentration of ATP during incubation (Means \& Hall, 1968). The results of this investigation employing an isolated mitochondrial fraction with tracer quantities of the substrate confirm both the high rate of ATP utilization in this organ, as well as the time course followed. Both ADP and AMP are formed. Approximately $92 \%$ of the total radioactivity recovered following $30-\mathrm{min}$ incubation is present in these two nucleotides. Similar experiments carried out with mitochondrial fractions derived from liver and kidney show less catabolism to ADP and AMP. This may be related to the fact that both liver (Atkinson \& Walton, 1967) and kidney (Chance \& Hess, 1959) have been reported to possess regulatory processes for the maintenance of a steady-state ATP concentration.

In view of the rapid catabolism of ATP by mitochondria from rat testis, it would seem apparent that the production of adequate levels of ATP, primarily through glucose metabolism, would be mandatory for the normal function of the organ. The demand for this energy-rich nucleotide is undoubtedly large in the testis in view of the fact that the germinal epithelium is a rapidly proliferating, protein-synthesizing tissue. Hence, any factor(s) which would interfere with the normal availability of intracellular ATP in the body could be expected to arrest the normal proliferative sequence of the testis as one of its early effects, in that the testis appears to degrade ATP more rapidly than other organs such as liver and kidney.

This investigation was supported by General Research Support Grant RR-0568-02 from the General Research Support Branch Division of Research Resources, National Institutes of Health.

The author would like to express his appreciation to Mr Robert Munn of the Health Sciences Advancement Award for the electron photomicrograph.

\section{REFERENCES}

Atkinson, D. E. \& WaLton, G. M. (1967) Adenosine triphosphate conservation in metabolic regulation. 7. biol. Chem. 242, 3239.

Chance, B. \& Hess, B. (1959) Metabolic control mechanisms. III. Kinetics of oxygen utilization in ascites tumor cells. F. biol. Chem. 234, 2416.

Hollinger, M. A. (1970) Studies on adenyl cyclase in rat testis. Life Sci. 9, 533.

Lowry, O. H., Rosebrough, N. J., Farr, A. L. \& Randall, R. J. (1951) Protein measurements with the folin phenol reagent. J. biol. Chem. 193, 265.

Means, A. R. \& Hall, P. J. (1968) Protein biosynthesis in the testis. II. Role of adenosine triphosphate (ATP) in stimulation by glucose. Endocrinology, 83, 86. 